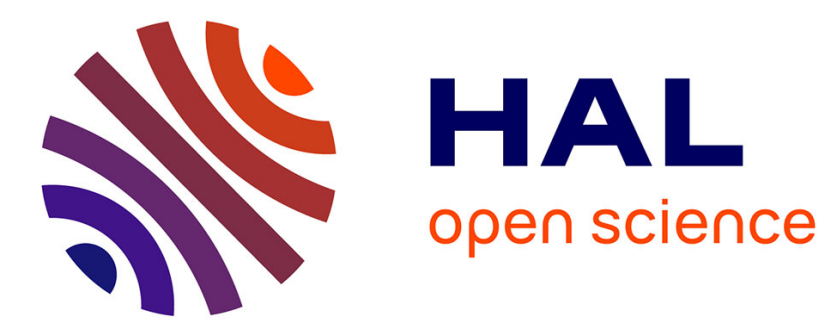

\title{
SOT-MRAM based Analog in-Memory Computing for DNN inference
}

\author{
J. Doevenspeck, Kevin Garello, B. Verhoef, R. Degraeve, S. van Beek, D. \\ Crotti, F. Yasin, S. Couet, G. Jayakumar, I. A Papistas, et al.
}

\section{- To cite this version:}

J. Doevenspeck, Kevin Garello, B. Verhoef, R. Degraeve, S. van Beek, et al.. SOT-MRAM based Analog in-Memory Computing for DNN inference. 2020 IEEE Symposium on VLSI Technology, Jun 2020, Honolulu (virtual), United States. 10.1109/VLSITechnology18217.2020.9265099 . hal03239881

\section{HAL Id: hal-03239881 \\ https://hal.science/hal-03239881}

Submitted on 27 May 2021

HAL is a multi-disciplinary open access archive for the deposit and dissemination of scientific research documents, whether they are published or not. The documents may come from teaching and research institutions in France or abroad, or from public or private research centers.
L'archive ouverte pluridisciplinaire HAL, est destinée au dépôt et à la diffusion de documents scientifiques de niveau recherche, publiés ou non, émanant des établissements d'enseignement et de recherche français ou étrangers, des laboratoires publics ou privés. 


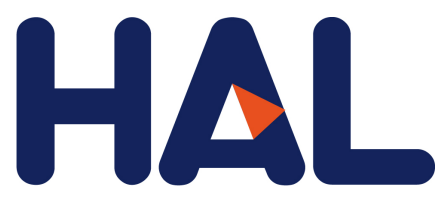

archives-ouvertes

\title{
SOT-MRAM based Analog in-Memory Computing for DNN inference
}

\author{
J. Doevenspeck, Kevin Garello, B. Verhoef, R. Degraeve, S. van Beek, D. \\ Crotti, F. Yasin, S. Couet, G. Jayakumar, I. Papistas, et al.
}

\section{To cite this version:}

J. Doevenspeck, Kevin Garello, B. Verhoef, R. Degraeve, S. van Beek, et al.. SOT-MRAM based Analog in-Memory Computing for DNN inference. 2020 IEEE Symposium on VLSI Technology, Jun 2020, Honolulu, France. pp.1-2, 10.1109/VLSITechnology18217.2020.9265099 • hal-03239881

\section{HAL Id: hal-03239881 \\ https://hal.archives-ouvertes.fr/hal-03239881}

Submitted on 27 May 2021

HAL is a multi-disciplinary open access archive for the deposit and dissemination of scientific research documents, whether they are published or not. The documents may come from teaching and research institutions in France or abroad, or from public or private research centers.
L'archive ouverte pluridisciplinaire HAL, est destinée au dépôt et à la diffusion de documents scientifiques de niveau recherche, publiés ou non, émanant des établissements d'enseignement et de recherche français ou étrangers, des laboratoires publics ou privés. 


\title{
SOT-MRAM based Analog in-Memory Computing for DNN inference
}

\author{
J. Doevenspeck ${ }^{1,2}$, K. Garello ${ }^{1}$, B. Verhoef ${ }^{1}$, R. Degraeve ${ }^{1}$, S. Van Beek ${ }^{1}$, D. Crotti ${ }^{1}$, F. Yasin ${ }^{1}$, S. Couet ${ }^{1}$, G. Jayakumar \\ I. A Papistas ${ }^{1}$, P. Debacker ${ }^{1}$, R. Lauwereins ${ }^{1,2}$, W. Dehaene ${ }^{1,2}$, G.S. Kar ${ }^{1}$, S. Cosemans ${ }^{1}$, A. Mallik ${ }^{1}$ and D. Verkest ${ }^{1}$ \\ ${ }^{1}$ imec, Leuven, Belgium, ${ }^{2} \mathrm{KU}$ Leuven ESAT, Leuven, Belgium, email: jonas.doevenspeck@imec.be
}

\begin{abstract}
Deep neural network (DNN) inference requires a massive amount of matrix-vector multiplications which can be computed efficiently on memory arrays in an analog fashion. This approach requires highly resistive memory devices $(>M \Omega)$ with low resistance variability to implement DNN weight memories. We propose an optimized Spin-Orbit Torque MRAM (SOTMRAM) as weight memory in Analog in-Memory Computing (AiMC) systems for DNN inference. In SOT-MRAM the write and read path are decoupled. This allows changing the MTJ resistance to the high levels required for AiMC by tuning the tunnel barrier thickness without affecting the writing.

The target resistance level and variation are derived from an algorithm driven design-technology-co-optimization (DTCO) study. Resistance levels are obtained from IR-drop simulations of a convolutional neural network (CNN). Variation limits are obtained by testing two noise-resilient CNNs with conductance variability. Finally, we demonstrate experimentally that the requirements for analog DNN inference are met by SOT-MRAM stack optimization.
\end{abstract}

Introduction: DNNs are built using mainly two types of layers: fully connected (FC) and convolutional (CONV) layers. The core operations in both layers are matrix-vector multiplications (MVMs) which can be mapped (Fig. 1) one-to-one to a crossbar array with programmable resistors (referred to as weight memory devices). The vector-matrix product can then be calculated in the analog domain using Ohm's and Kirchhoff's law. This AiMC [1] approach avoids costly memory fetches from an external memory and is more energy-efficient than digital multiply-accumulate (MAC) circuits [2].

Although DNN training requires many weight levels (more than 256 [3]), quantized DNNs for inference can achieve top accuracy using ternary weight levels [4]. A correct AiMC implementation depends on memory devices having stable $>\mathrm{M} \Omega$ resistance levels and low resistance variability. MRAM is one of the most promising candidates fulfilling these requirements.

The standard writing mechanism in MRAM technology, spin transfer torque (STT), cannot be used to switch high resistance MTJs, contrary to the SOT writing mechanism (Fig. 2). In this work, we demonstrate experimentally that SOT-MRAM is an excellent candidate to implement ternary weights for inference accelerators running quantized DNNs. Also, we develop a DTCO analysis to guide the process optimisation to make SOT-MRAM suitable for this application

Experimental demonstration: Fig. 3 shows a TEM of a SOT-MTJ cell integrated on $300 \mathrm{~mm}$ wafers using CMOScompatible processes. As sketched in Fig.2, the in-plane write current flows from bottom electrode 1 (BE1) to BE2 while the reading current is probed from the top electrode (TE) to BE2. The device resistance of the SOT-MTJ cell can be tuned through the $\mathrm{MgO}$ thickness as shown in Fig. 4. The SOT device is based on $[5,6]$. However, directly adapting this SOT stack with an increased $\mathrm{MgO}$ thickness results in severe coercivity $\left(\mathrm{B}_{\mathrm{c}}\right)$ loss, $\mathrm{B}_{\mathrm{c}}$ being a direct evaluation of device retention. Therefore, the stack has been reworked and optimized in order to recover sufficient coercivity at large RA products (Fig. 5). This is exemplified in Fig. 6 by R-H loops for devices with nominal CD $(\mathrm{nCD})=80 \mathrm{~nm}$ and $\mathrm{RA}=5,20,50 \mathrm{k} \Omega \cdot \mu \mathrm{m}^{2}$.

Next, we applied $0.5 \mathrm{~ns}$ voltage pulses across the SOT-track to switch the same $80 \mathrm{~nm}$ devices, and we confirm as expected that highly resistive SOT-MTJ cells do not compromise SOTswitching performance (Fig. 7). Finally, we evaluated the resistance statistics for different MTJ RAs and CDs (Fig. 8a). Note that these numbers are obtained from a device test vehicle and do not represent the fundamental lower limit of resistance variability. Importantly, we observe that the coefficient of variation $(\sigma / \mu)$ does not increase for increasing RA products (Fig. $8 \mathrm{~b})$. The MTJ resistance spread is indeed mostly determined by process-induced area variations (Fig. 8c), which can be fully optimized at production level. In the next section, we investigate the impact of resistance variation on DNNs over a wide range, with the measured variation as reference point.

\section{Targets for resistance levels and variability:}

Assuming a differential conductance pair weight encoding scheme (Fig. 9), the relation between device conductance variability and DNN weight variability can be derived (Fig. 10). Since a weight 0 is mapped on two devices in the AP state, this puts a constraint on the AP state distributions. The weights -1 and 1 are mapped on one $\mathrm{P}$ and one AP state. So, they put a constraint on both the P and AP state distributions. The tolerated $\sigma / \mu$ of the conductance state distributions for both the 0 and $-1,1$ weights is given in Fig. 11. Two networks are used to derive the specifications for the weight memory device. Both networks are used to explore the tolerance against weight variation induced by conductance variations.

Analog inference accelerators add two specific challenges: variation on the network weights and quantization to a very limited amount of activation and weight levels. To avoid accuracy loss due to this mapping, the training procedure needs to be adapted (Fig. 12). Fig 13. shows the test error for CNN 1 on MNIST after training with and without variation added to the weights. Here, the same $\sigma_{\text {weight }}\left(\sigma_{\mathrm{W}}\right)$ is used during testing $\left(\sigma_{\mathrm{W}, \text { test }}\right)$ as during training $\left(\sigma_{\mathrm{W}, \text { train }}\right)$. Training with weight variation clearly decreases the test error for the whole range of weight variation. The same experiment was repeated for CNN 2 on CIFAR100 (Fig 14). However, to explore the tolerance of the network, each trained network was tested with a range of different $\sigma_{\mathrm{W} \text {,test }}$ values. Interestingly, training with $\sigma_{\mathrm{W} \text {,train }}>\sigma_{\mathrm{W} \text {,test }}$ yields a better accuracy than $\sigma_{\mathrm{W} \text {,train }}=\sigma_{\mathrm{W} \text {,est. }}$ This means that an aggressively trained network can be deployed on a variety of analog inference

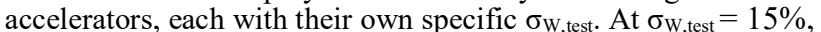
the error increase is limited to $1.5 \%$. Given that CIFAR 100 is a representative benchmark for real-world applications and assuming $1.5 \%$ error increase is acceptable, $\sigma_{\mathrm{W}, \text { test }}<15 \%$ is used as target for weight variation.

Next, we evaluate the impact of the wire resistance $\mathrm{R}_{\text {WIRE }}$ on the network accuracy on the entire test set (10.000 examples). A fully parallel GPU-compatible IR-drop solver performs a DC simulation for each analog MVM and assumes all other operations such as pooling, ReLU, BatchNorm, etc. are performed errorless in the digital domain (Fig. 15a). An example of an IR-drop simulation is given in Fig. 15b. Weights further away from the activation/summation line voltage source see a lower voltage. Hence, they draw less current and contribute too little to the matrix-vector multiplication product, giving rise to a reduced effective weight. Fig. 16 shows the DC simulations results for $\mathrm{CNN}$ inference using the line resistance extracted from the layout in Fig. 9 and two other reference points. Below an on resistance $\left(\mathrm{R}_{\mathrm{ON}}\right)$ of $5 \mathrm{M} \Omega$, the test error increases due to IR-drop.

In Table I, device metrics relevant for analog DNN inference systems are compared between SOT-MRAM and other popular devices. Our optimized SOT-MRAM devices at $\mathrm{nCD}=80 \mathrm{~nm}$ and $\mathrm{RA}=20 \mathrm{k} \Omega . \mu \mathrm{m}^{2}$ meet the $\mathrm{R}_{\mathrm{ON}}>5 \mathrm{M} \Omega$ and $\sigma_{\mathrm{W}}<15 \%$ requirement while maintaining a low write voltage $\left(\mathrm{V}_{\mathrm{WR}}\right)$.

Conclusions: Using the SOT writing mechanism, we demonstrated for the first time that highly resistive MRAM devices can be used as weight memory for AiMC DNN inference. We conducted a DTCO study where CNNs (for MNIST and CIFAR100) are trained and tested including resistance variation and the effect of wire IR-drop. It shows that SOT-MRAM meets the derived specifications.

Acknowledgments: This research is conducted within the imec IIAP entitled "Machine Learning". This project has received funding from the Electronic Components and Systems for European Leadership Joint Undertaking under grant agreement No 826655 .

References: [1] S. Cosemans et al., IEDM 2019 [2] D. Bankmann et al., JSSCC, vol. 54 no. 1 p. 158 (2018) [3] N. Wang et al., NIPS, p. 7686 (2018) [4] J. Choi et al., SysML, (2019) [5] K. Garello et al., VLSI, p. 81 (2018) [6] K. Garello et al., VLSI, JFS4-5 (2019) [7] LeCun et al., Proc. of the IEEE, vol. 86 no. 11 p. 2278 (1998) [8] K. He et al., CVPR, p. 770 (2015) 


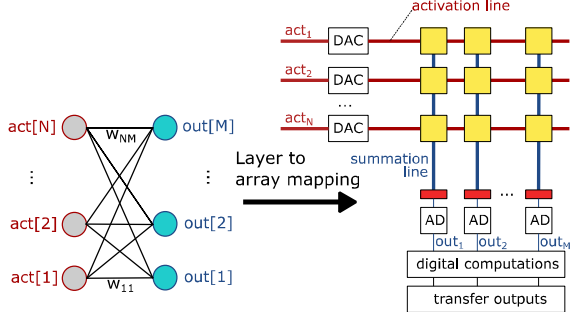

Fig.1: ACIM concept: DNN layer to analog matrixvector-multiply (MVM) mapping. The matrix-vector

product can be calculated in the analog domain using
Ohm's and Kirchhoff's law.

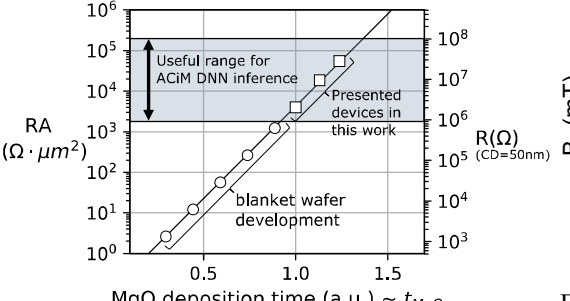

MgO deposition time (a.u.) $\sim t_{M g O}$

Fig. 4 The MTJ RA product scales exponentially with $\mathrm{MgO}$ thickness which allows to easily DNN inference.

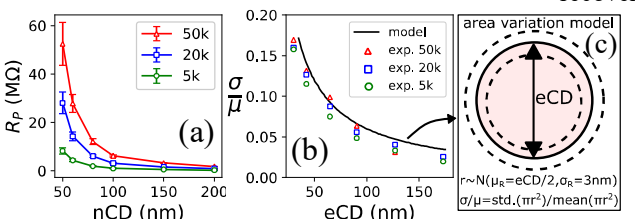

Fig. 8 a) Parallel resistance $\left(R_{P}\right)$ statistics for different MTJ CDs and RA products. b) $\sigma / \mu$ for different electrical CDs (eCD) and RA. (c) Resistance variation can be attributed to MTJ area variation. A gaussian distribution on the MTJ radius with $\sigma=3 \mathrm{~nm}$ sufficies to capture nearly all variation.

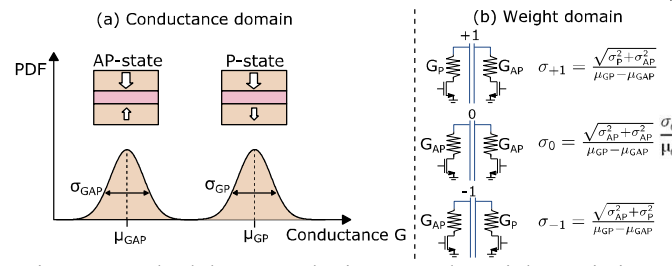

Fig. 10 Methodology to obtain network weight variation from measured conductance distributions. (a) AP and P conductance distributions. (b) Standard deviation of the weight levels $\sigma_{-1,0,+1}$ depends on $\sigma_{\mathrm{GAP}}, \sigma_{\mathrm{GP}}$ and $\mu_{\mathrm{GP}}-\mu_{\mathrm{GAP}}$. (a) inputs

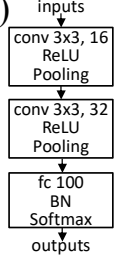

(b) 3

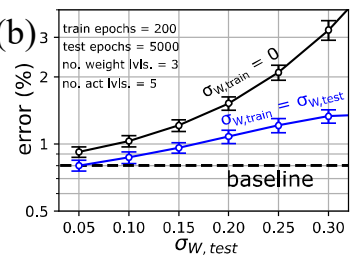

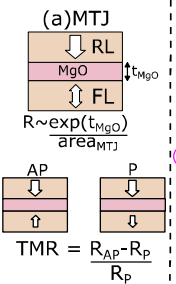

Fig. 2. (a) Magnetic tunnel junction (MTJ) (b) Threerite Therefore, the RA product is not limited by the SOTspin-current perpendicular to the free layer (FL) (b) SOT-MRAM

$\mathrm{V}_{\text {READ }}$ STT-Writt

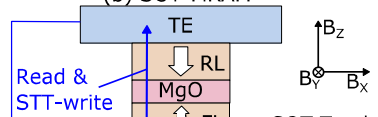

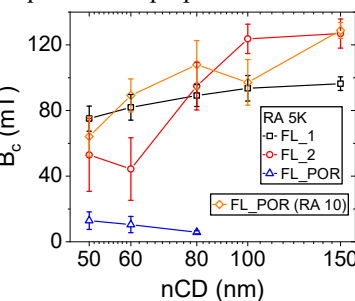

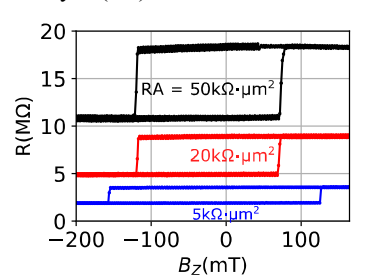

Fig. 6 R-H loops as function of Fig. 7 R-V switching for a device with

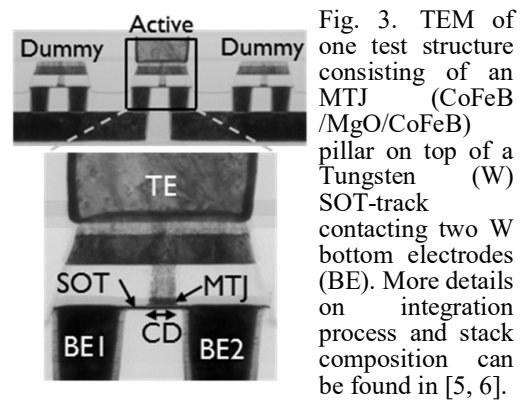

Fig. 5 Stack optimization to retain external $\mathrm{Z}$ field $\mathrm{B}_{\mathrm{Z}}$ for devices $\mathrm{nCD}=80 \mathrm{~nm}$ and various $\mathrm{RAs}$. SOTStack engineering the FL increasing RA products is fully compatible with our AIMC approach.

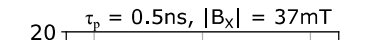

\section{.}
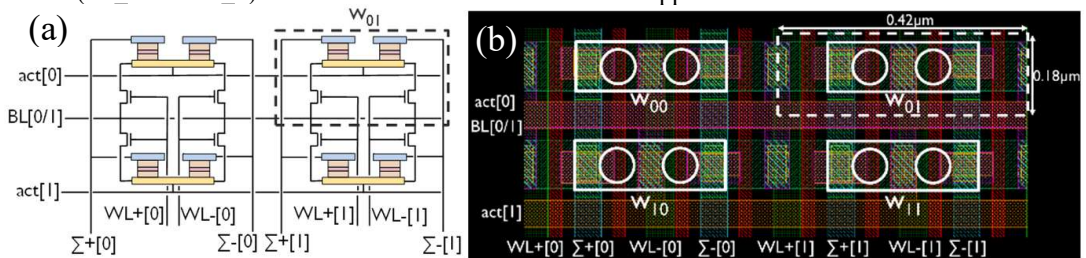

Fig 9: Schematic (a) and layout (b) of $2 \times 2$ array. Each weight is mapped to a differential conductance pair. The layout (made with a commercial $22 \mathrm{~nm}$ PDK) is dimensioned for SOT-MRAM devices with pillar diameter $\leq 80 \mathrm{~nm}$ and uses one two finger transistor for each SOT-MRAM device. By sharing one SOT-track with two pillars, contact area is reduced. The area of one ternary compute cell is $0.076 \mu \mathrm{m}^{2}$.

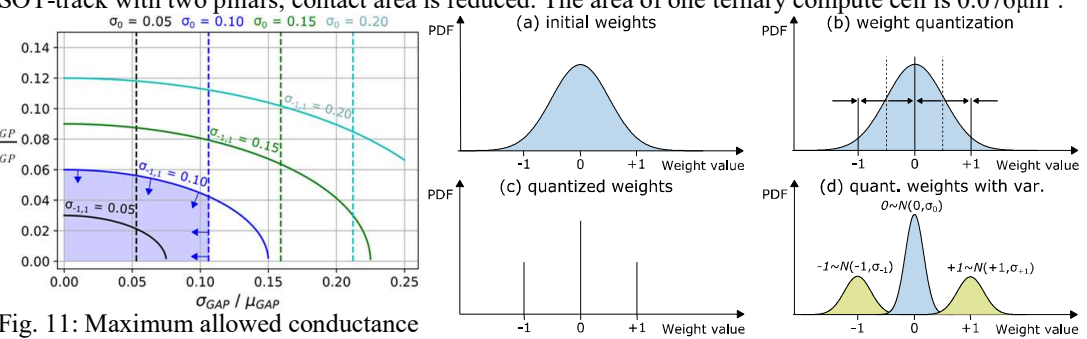

Fig. 11: Maximum allowed conductance distribution variation for given weight Fig. 12: During each forward pass in the training, the floating variation $\sigma_{\mathrm{W}}$ for both $-1,0,+1$ weights. point weights are quantized and weight variation $\sigma_{\mathrm{W} \text {,train }}$ is TMR is assumed to be $150 \%$.
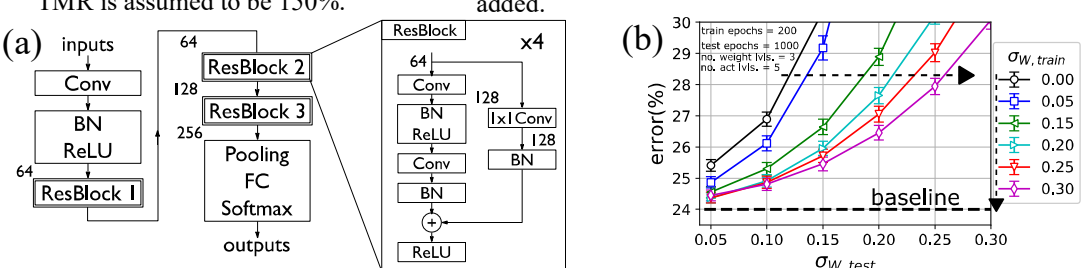

Fig. 13. (a) CNN 1 inspired by LeNet-5 [7] to achieve Fig. 14. (a) ResNet-26 CNN 2 [8] used to train and test CIFAR 100 with weight variation. The network is $99.2 \%$ accuracy on MNIST. The network is used to sized to achieve $76 \%$ accuracy. (b) Test error on CIFAR 100 after training with different $\sigma_{\mathrm{W}, \text { train }}$. Up to $\sigma_{\mathrm{W}, \text { test }}=$ train and test with weight variation and in IR-drop 0.15 , the error increase is $<1.5 \%$ by using $\sigma_{\mathrm{W} \text {,train }}=0.30$. In general, using a $\sigma_{\mathrm{W} \text {,train }}>\sigma_{\mathrm{W} \text {,test }}$ results in smaller simulations to set resistance level targets. (b) Test error increase compared to using $\sigma_{\mathrm{W} \text {,train }}=\sigma_{\mathrm{W} \text {,test. }}$. Increasing $\sigma_{\mathrm{W} \text {,train }}$ beyond 0.30 increases the error at low $\sigma_{\mathrm{W}, \text { test }}$ error on MNIST for different $\sigma_{\mathrm{W} \text {,train }}$ and $\sigma_{\mathrm{W} \text {,test. }} \quad$ so is not desirable (not shown on this figure).
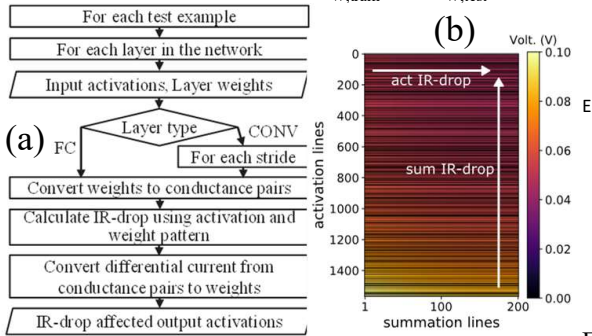

Fig. 15 (a) Methodology to assess impact of IR-drop on network accuracy. (b) Example of IR-drop in the FC layer of CNN 1. IR-drop happens both along the activation and summation line.

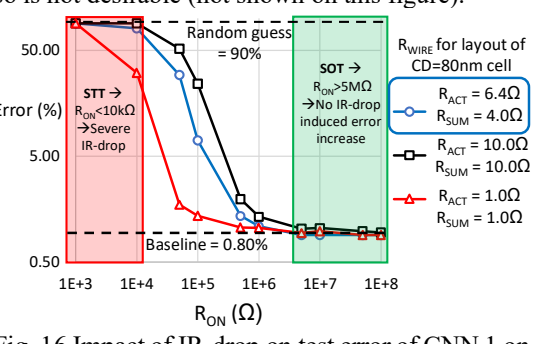

Fig. 16 Impact of IR-drop on test error of CNN 1 on MNIST for different $R_{O N}$ and $R_{\text {WIRE. }} R_{O F F}$ is recovered at $\mathrm{R}_{\mathrm{ON}}>5 \mathrm{M} \Omega$.

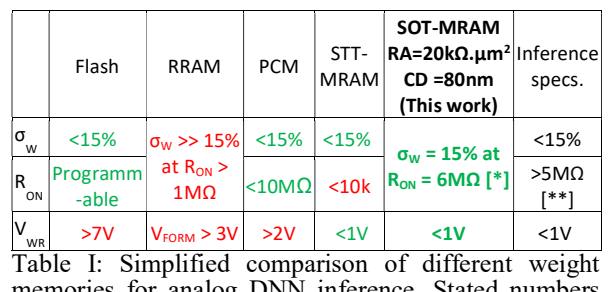
represent typical operating conditions and are not fundamental lower limits. [*] Assuming TMR $=150 \%$. $[* *]$ This specification depends on array size and wire resistance but is representative for relevant AiMC array sizes ( $>1000$ rows) and sub $22 \mathrm{~nm}$ nodes. 